\title{
Corrigendum to "Multifractal-Multiscale Analysis of Cardiovascular Signals: A DFA-Based Characterization of Blood Pressure and Heart-Rate Complexity by Gender"
}

\author{
Paolo Castiglioni $\mathbb{D}^{1},{ }^{1}$ Davide Lazzeroni, ${ }^{1}$ Paolo Coruzzi, ${ }^{2}$ and Andrea Faini ${ }^{3}$ \\ ${ }^{1}$ IRCCS Fondazione Don C. Gnocchi, Milan, Italy \\ ${ }^{2}$ Department of Medicine and Surgery, University of Parma, Parma, Italy \\ ${ }^{3}$ Department of Cardiology, Istituto Auxologico Italiano, Milan, Italy \\ Correspondence should be addressed to Paolo Castiglioni; paolo.castiglioni@gmail.com \\ Received 26 March 2018; Accepted 3 April 2018; Published 30 April 2018
}

Copyright (c) 2018 Paolo Castiglioni et al. This is an open access article distributed under the Creative Commons Attribution License, which permits unrestricted use, distribution, and reproduction in any medium, provided the original work is properly cited.

\begin{abstract}
In the article titled "Multifractal-Multiscale Analysis of Cardiovascular Signals: A DFA-Based Characterization of Blood Pressure and Heart-Rate Complexity by Gender" [1], the affiliation of the second author was given incorrectly. The correct affiliation is shown above.
\end{abstract}

\section{References}

[1] P. Castiglioni, D. Lazzeroni, P. Coruzzi, and A. Faini, "Multifractal-Multiscale Analysis of Cardiovascular Signals: A DFA-Based Characterization of Blood Pressure and Heart-Rate Complexity by Gender," Complexity, vol. 2018, Article ID 4801924, 14 pages, 2018. 


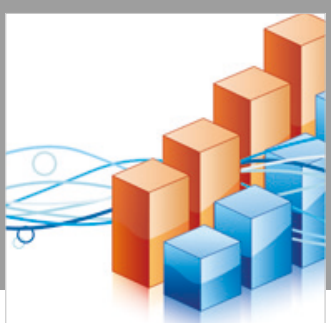

Advances in

Operations Research

\section{-n-m}
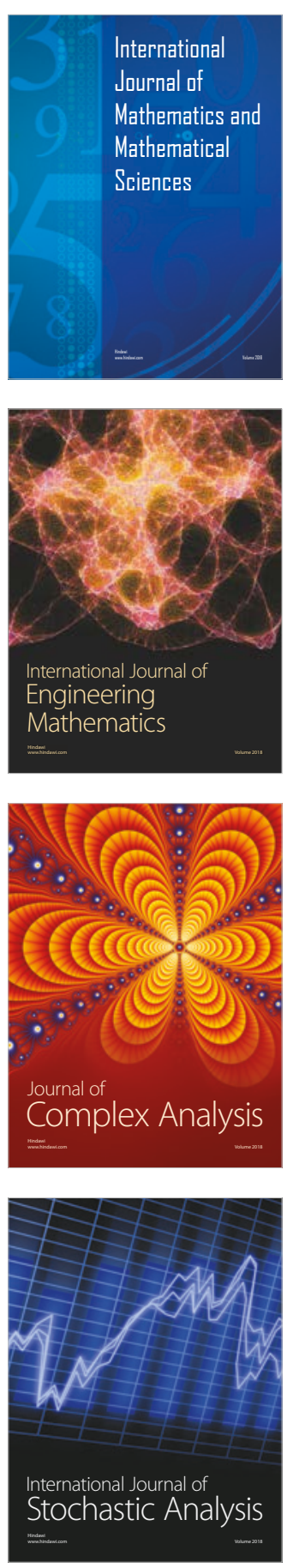
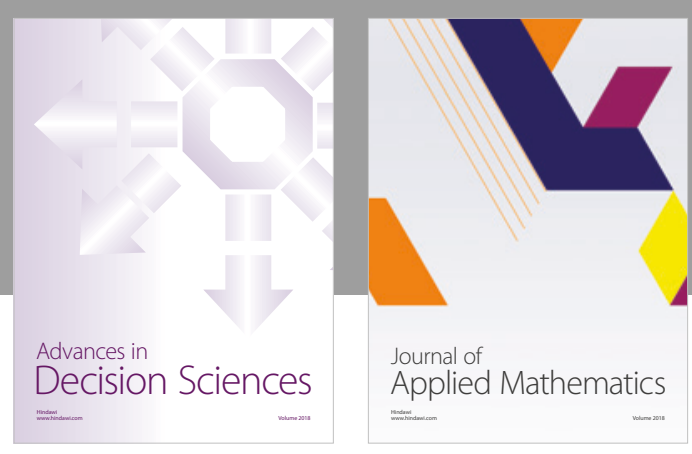

Journal of

Applied Mathematics
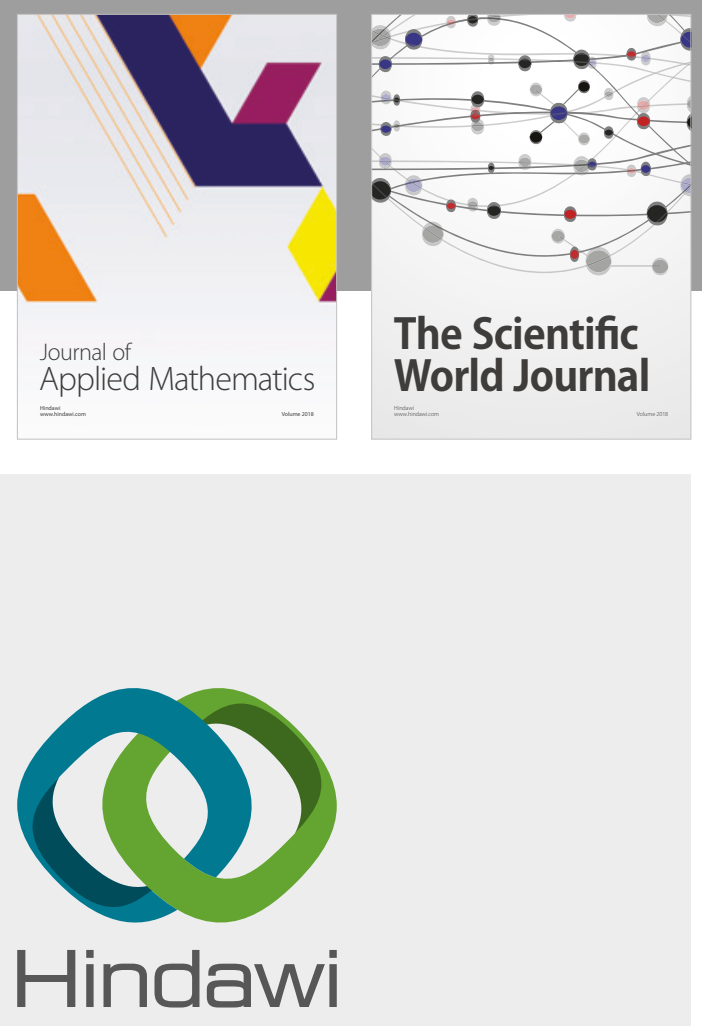

Submit your manuscripts at

www.hindawi.com

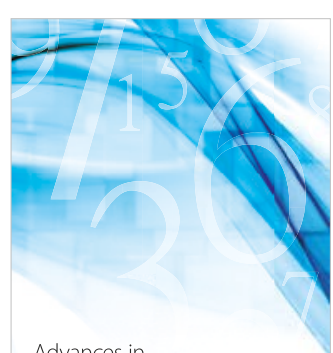

Advances in
Numerical Analysis
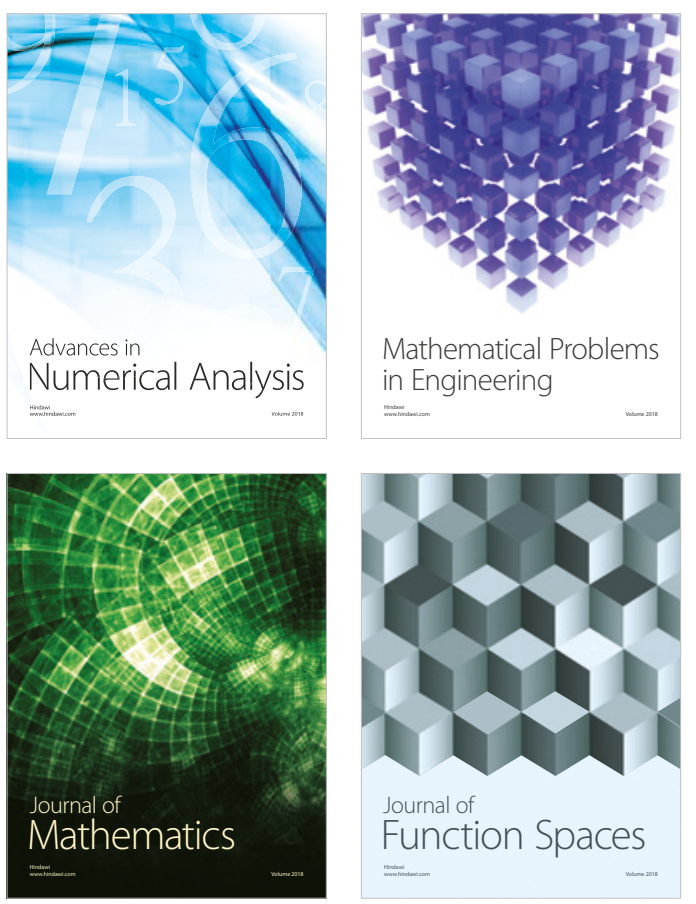

Mathematical Problems in Engineering

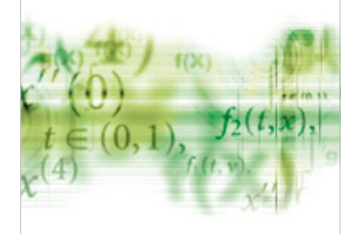

International Journal of

Differential Equations

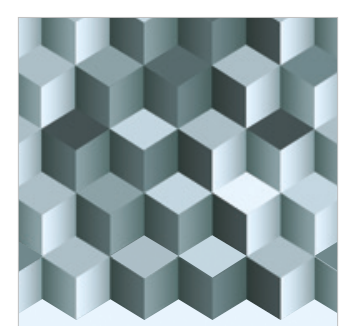

Journal of

Function Spaces
The Scientific

World Journal

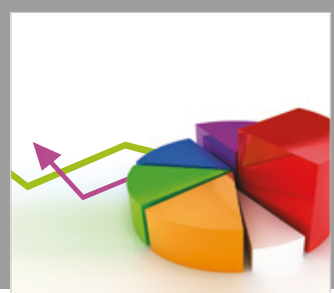

Journal of

Probability and Statistics
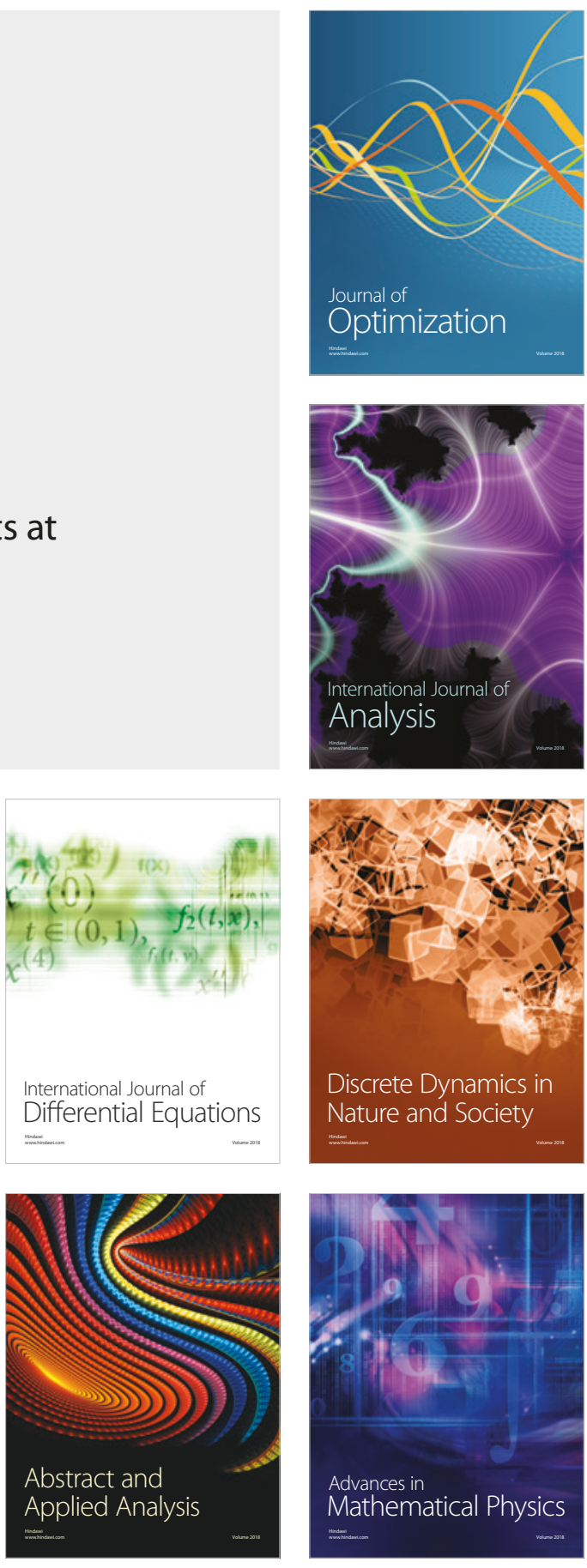